\title{
Knockdown of KIAA1199 attenuates growth and metastasis of hepatocellular carcinoma
}

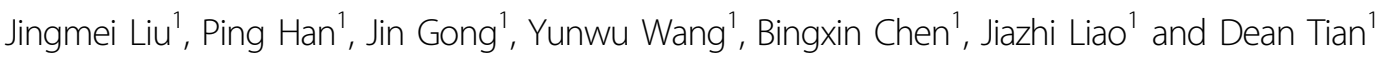

\begin{abstract}
Accumulating evidence indicates that KIAA1199 plays a vital role in tumor progression. However, the role of KIAA1199 in hepatocellular carcinoma (HCC) still remains unknown. In this study, we found that KIAA1199 was upregulated in human HCC tissues and in highly metastatic HCC cell lines. Furthermore, the expression of KIAA1199 was significantly correlated with tumor size and metastasis in HCC. Knockdown of KIAA1199 inhibited cell proliferation and migration in vitro, and suppressed tumorigenicity and lung metastasis in vivo. In addition, silencing of KIAA1199 induced G1 phase arrest by reducing cyclinD1 expression. Moreover, KIAA1199 knockdown induced apoptosis by activating endoplasmic reticulum (ER) stress, which was based on the upregulation of ER stress markers, activating transcription factor 4 (ATF4) and CAAT/enhancer-binding protein homologous protein (CHOP). In conclusion, our data demonstrated that KIAA1199 knockdown inhibited the growth and metastasis of HCC.
\end{abstract}

\section{Introduction}

Hepatocellular carcinoma (HCC) is the third leading cause of cancer-related mortality worldwide ${ }^{1}$. More than $90 \%$ of cancer-related deaths are the consequence of the tumor cells escaped from primary tumor to form metastases $^{2}$. Although genetic and epigenetic alterations are well characterized in HCC, the molecular pathogenesis still remains largely unknown ${ }^{3}$. Thus, additional studies must be carried out to gain a better understanding of HCC progression in order to develop novel therapeutic targets.

KIAA1199 was first identified as an inner ear-specific protein, which is located on chromosome band $15 \mathrm{q} 25.1^{4}$. It has been reported that KIAA1199 is upregulated in many types of tumors, such as colorectal cancer, gastric cancer, and breast cancer ${ }^{5-7}$. Moreover, high levels of KIAA1199 were correlated with worse five-year survival

\footnotetext{
Correspondence: Jiazhi Liao (liaojiazhi2011@163.com) or Dean Tian (datian@tjh.tjmu.edu.cn)

'Department of Gastroenterology, Tongji Hospital, Tongji Medical College, Huazhong University of Science and Technology, Wuhan, China Edited by A. E. Sayan
}

outcomes in colon cancer ${ }^{8}$ and lymph node metastasis in gastric cancer ${ }^{7}$. Evensen et al. found that KIAA1199mediated cell migration relies on $\mathrm{ER} \mathrm{Ca}^{2+}$ leakage, followed by the protein kinase $\mathrm{C}-\alpha$ isoform (PKC $\alpha$ ) activation $^{9}$. These findings are consistent with a recent report, which showed that repression of KIAA1199 decreased the proliferation and invasion of colon cancer cells by modulating $\mathrm{Ca}^{2+}$ signaling ${ }^{10}$. Together, these results strongly suggest a significant role of KIAA1199 in tumor progression through diverse mechanisms. However, the exact function of KIAA1199 in HCC has not been investigated yet.

In the present study, we demonstrated that KIAA1199 was upregulated in HCC tissues, and the expression of KIAA1199 was positively correlated with the metastatic potentials of HCC cells. Furthermore, we also confirmed that KIAA1199 knockdown inhibited the growth and metastasis of HCC by both in vitro and in vivo assays. Mechanistic studies revealed that KIAA1199 knockdown induced cell apoptosis by activating endoplasmic reticulum (ER) stress. Moreover, these findings provided novel mechanistic insights into the function of KIAA1199.

\section{(c) 2018 The Author(s).}

(c) Open Access This article is licensed under a Creative Commons Attribution 4.0 International License, which permits use, sharing, adaptation, distribution and reproduction cc) in any medium or format, as long as you give appropriate credit to the original author(s) and the source, provide a link to the Creative Commons license, and indicate if changes were made. The images or other third party material in this article are included in the article's Creative Commons license, unless indicated otherwise in a credit line to the material. If material is not included in the article's Creative Commons license and your intended use is not permitted by statutory regulation or exceeds the permitted use, you will need to obtain permission directly from the copyright holder. To view a copy of this license, visit http://creativecommons.org/licenses/by/4.0/. 


\section{Results}

Expression and clinicopathologic significance of KIAA1199 in $\mathrm{HCC}$

To explore the effect of KIAA1199 on HCC, RT-qPCR and immunohistochemistry was performed to determine the expression of KIAA1199 in 64 pairs of HCC samples. Compared with the corresponding pericarcinoma tissues, KIAA1199 was significantly upregulated in 47 pairs of HCC tissues (73.44\%) (Fig. 1a), and staining of KIAA1199 in HCC samples showed positivity mostly in the cytoplasm (Fig. 1b). Further analysis of the clinicopathological characteristics in HCC samples showed that KIAA1199 overexpression positively correlated with tumor size $(P=$ $0.043)$ and metastasis $(P=0.023)$, but no significant differences were observed with respect to age, sex, tumor number, and the alpha-fetoprotein (AFP) level (Table 1). In addition, we also analyzed the expression of KIAA1199 in the normal liver cell line L02 and six HCC cell lines. In accord with the results from tissues, we found that KIAA1199 was overexpressed in highly invasive HCC cell lines and hardly any in the normal liver cell line (Fig. 1c, d). These results indicated that KIAA1199 played an important role in HCC progression.

\section{KIAA1199 knockdown inhibits HCC cell proliferation and migration}

To investigate the functional roles of KIAA1199 in HCC, KIAA1199 expression in metastatic HCC cell lines (HCCLM3 and MHCC-97H) was suppressed by RNA interference (RNAi). Successful knockdown of KIAA1199 was illustrated by RT-qPCR and western blotting (Fig. 2a, b). Initially, the capacity of colony formation was evaluated in HCC-LM3 and MHCC-97H cells that were transfected with si KIAA1199 or si control. Compared with the si control-transfected cells, cells transfected with si KIAA1199 had much fewer and smaller colonies (Fig. 2c). Next, the effect of KIAA1199 on cell proliferation was assessed by CCK- 8 assays. The results demonstrated that HCC-LM3 and MHCC-97H cells transfected with siKIAA1199 showed a lower proliferation rate than the control cells (Fig. 2d). Furthermore, transwell and wound healing assays revealed that silencing of KIAA1199 caused a suppression of cell

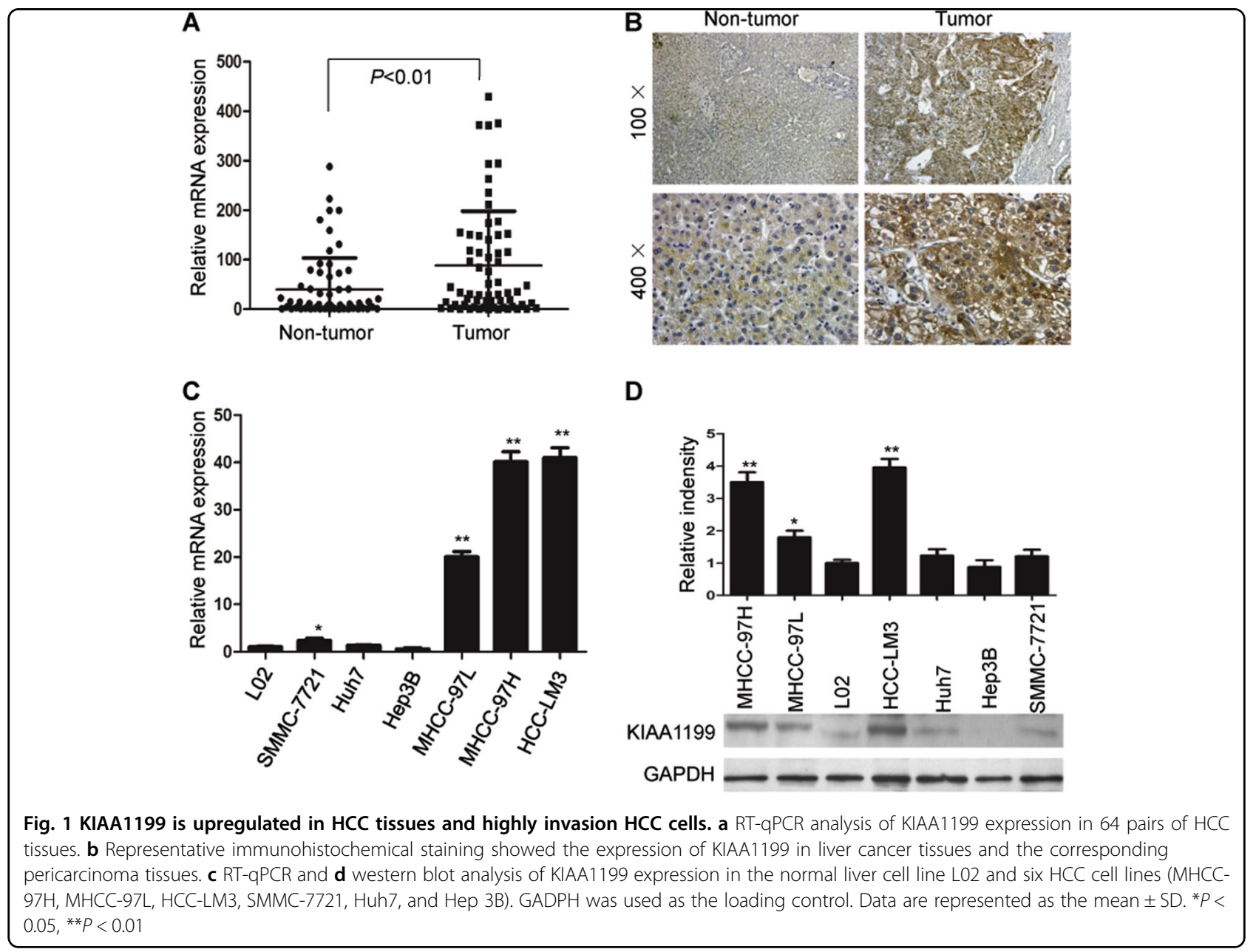


Table 1 KIAA1199 expression and clinicopathological factors

\begin{tabular}{|c|c|c|c|c|}
\hline \multirow[t]{2}{*}{ Variable } & & \multicolumn{2}{|l|}{ KIAA1199 } & \multirow[t]{2}{*}{$P$ value } \\
\hline & & $\begin{array}{l}\text { High } \\
\text { expression } \\
(n=47)\end{array}$ & $\begin{array}{l}\text { Low } \\
\text { expression } \\
(n=17)\end{array}$ & \\
\hline \multirow[t]{2}{*}{ Sex } & Male & 43 & 14 & 0.561 \\
\hline & Female & 4 & 3 & \\
\hline \multirow[t]{2}{*}{ Age (year) } & $\leq 45$ & 16 & 6 & 0.924 \\
\hline & $>45$ & 31 & 11 & \\
\hline \multirow[t]{2}{*}{$\mathrm{HBsAg}$} & Positive & 35 & 12 & 0.748 \\
\hline & Negative & 12 & 5 & \\
\hline \multirow[t]{2}{*}{ AFP (ng/ml) } & $\leq 400$ & 14 & 7 & 0.392 \\
\hline & $>400$ & 33 & 10 & \\
\hline $\begin{array}{l}\text { Tumor } \\
\text { diameter }\end{array}$ & $\leq 5$ & 10 & 8 & $0.043^{*}$ \\
\hline$(\mathrm{cm})$ & $>5$ & 37 & 9 & \\
\hline \multirow{2}{*}{$\begin{array}{l}\text { Tumor } \\
\text { number }\end{array}$} & Single & 38 & 11 & 0.182 \\
\hline & Multi & 9 & 6 & \\
\hline \multirow[t]{2}{*}{ Metastasis } & Yes & 34 & 7 & $0.023^{*}$ \\
\hline & No & 13 & 10 & \\
\hline
\end{tabular}

KIAA1199 high (low) expression (whose relative expression of HCC tissues was higher (lower) than that of corresponding pericarcinoma tissues according to the results of PCR). AFP alpha-fetoprotein

*Indicates statistical significance

migration in HCC-LM3 and MHCC-97H cells (Fig. 2e, f). Therefore, KIAA1199 knockdown inhibited HCC cell proliferation and migration.

\section{KIAA1199 knockdown suppresses the growth and metastasis of HCC in vivo}

Having observed that KIAA1199 played a critical role in promoting HCC cells proliferation and migration in vitro, we evaluate the function of KIAA1199 on tumor growth and metastasis by subcutaneous xenograft models and orthotopic xenograft models, respectively. HCC-LM3shRNA and HCC-LM3-shKIAA1199 cells were subcutaneously injected into nude mice, and tumor growth was assessed. Compared with the HCC-LM3-shRNA group, the sizes and weight of tumors in HCC-LM3shKIAA1199 group was decreased (Fig. 3a, b). In orthotopic xenograft models, tumor development was monitored by bioluminescent (BLI) imaging. BLI imaging revealed that the growth of tumors in HCC-LM3shKIAA1199 group was slower than the HCC-LM3-sh control group (Fig. 3c). Moreover, BLI imaging and $\mathrm{H} \& \mathrm{E}$ staining revealed significant lung metastasis in the HCC-
LM3-sh control group, while in the HCC-LM3shKIAA1199 group, little to no lung metastasis was detected (66.7\% versus 16.7\%) (Fig. 3d, e). Collectively, these results suggested that reduction of KIAA1199 inhibited HCC growth and metastasis in vivo.

\section{KIAA1199 knockdown induces G1 arrest by reducing cyclinD1}

Silencing of KIAA1199 suppressed the growth of HCC cells. To elaborate the mechanism of this inhibitor effect, we evaluated the cell cycle distribution of HCC cells by flow cytometry. Compared with the control cells, MHCC-97H and HCC-LM3 cells transfected with si KIAA1199 showed a significant decrease of the percentage of cells in the $S$ phase, and cell cycle was significantly blocked at the G1 checkpoint (Fig. 4a, b). As KIAA1199 knockdown caused cell cycle arrest in G1 phase, we investigated whether the expression of several key factors regulating G1/S cell cycle transition were affected. The results demonstrated that reduction of KIAA1199 decreased the level of cyclinD1, while the expression of cyclinE, cyclin-dependent kinases 2 (CDK2), and cyclin-dependent kinases 4 (CDK4) were not observably affected in both MHCC-97H (Fig. 4c, d) and HCC-LM3 cells (Fig. 4e, f). These data indicated that KIAA1199 knockdown induced cell cycle arrest by reducing cyclinD1 gene expression.

\section{KIAA1199 knockdown induces ER stress-mediated apoptosis}

Next, we explored the effect of KIAA1199 on cell apoptosis. Analysis of apoptosis by flow cytometry demonstrated that repression of KIAA1199 significantly increased the percentage of apoptotic cells in both HCCLM3 and MHCC-97H cells (Fig. 5a). We also detected the effect of KIAA1199 knockdown on cell apoptosis in HCCLM3 and MHCC-97H cells by TUNEL assay and caspase3 activity assay. Compared with the cells transfected with si control, si KIAA1199 treatment increased the number of TUNEL-positive cells (Fig. 5b). Expectedly caspase-3 activity was also induced in MHCC-97H and HCC-LM3 cells after KIAA1199 silencing (Fig. 5c). Accumulating evidences indicate that persistent and excessive ER stress can cause an apoptotic response ${ }^{11}$. Activating transcription factor 4 (ATF4) and CAAT/enhancer-binding protein homologous protein (CHOP) are the key signal molecules that lead to ER stress-mediated apoptosis ${ }^{12}$. To determine whether ER stress played a role in KIAA1199 knockdowninduced apoptosis, the levels of ER stress-associated markers were detected in HCC cells transfected with siRNAs (si control or si KIAA1199). The results showed that silencing of KIAA1199 significantly induced the expression of CHOP and ATF4, but had no impact on the expression of glucose-regulated protein, $78 \mathrm{kDa}$ (Grp-78, $\mathrm{BiP})$, which has a positive regulatory role in the 


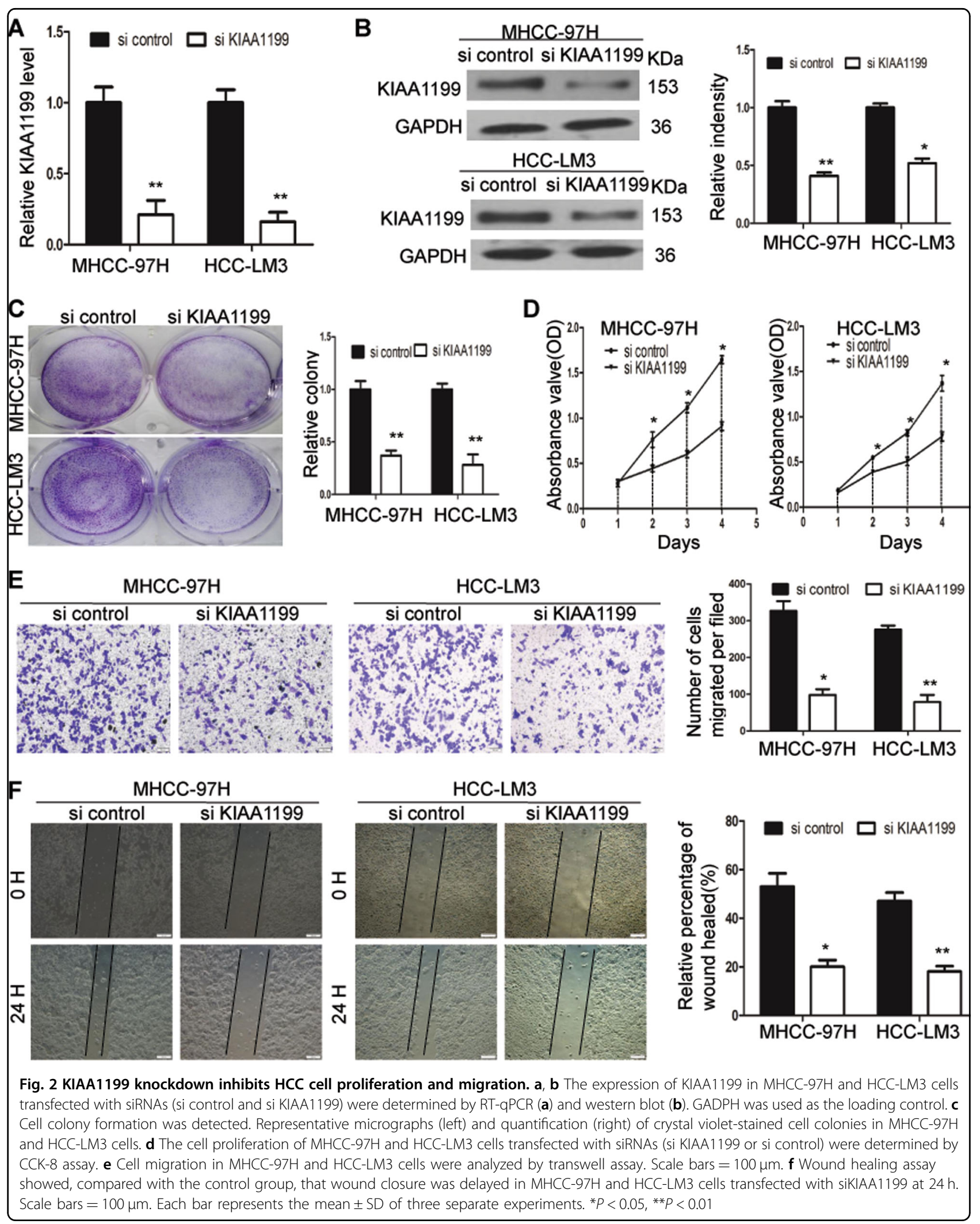




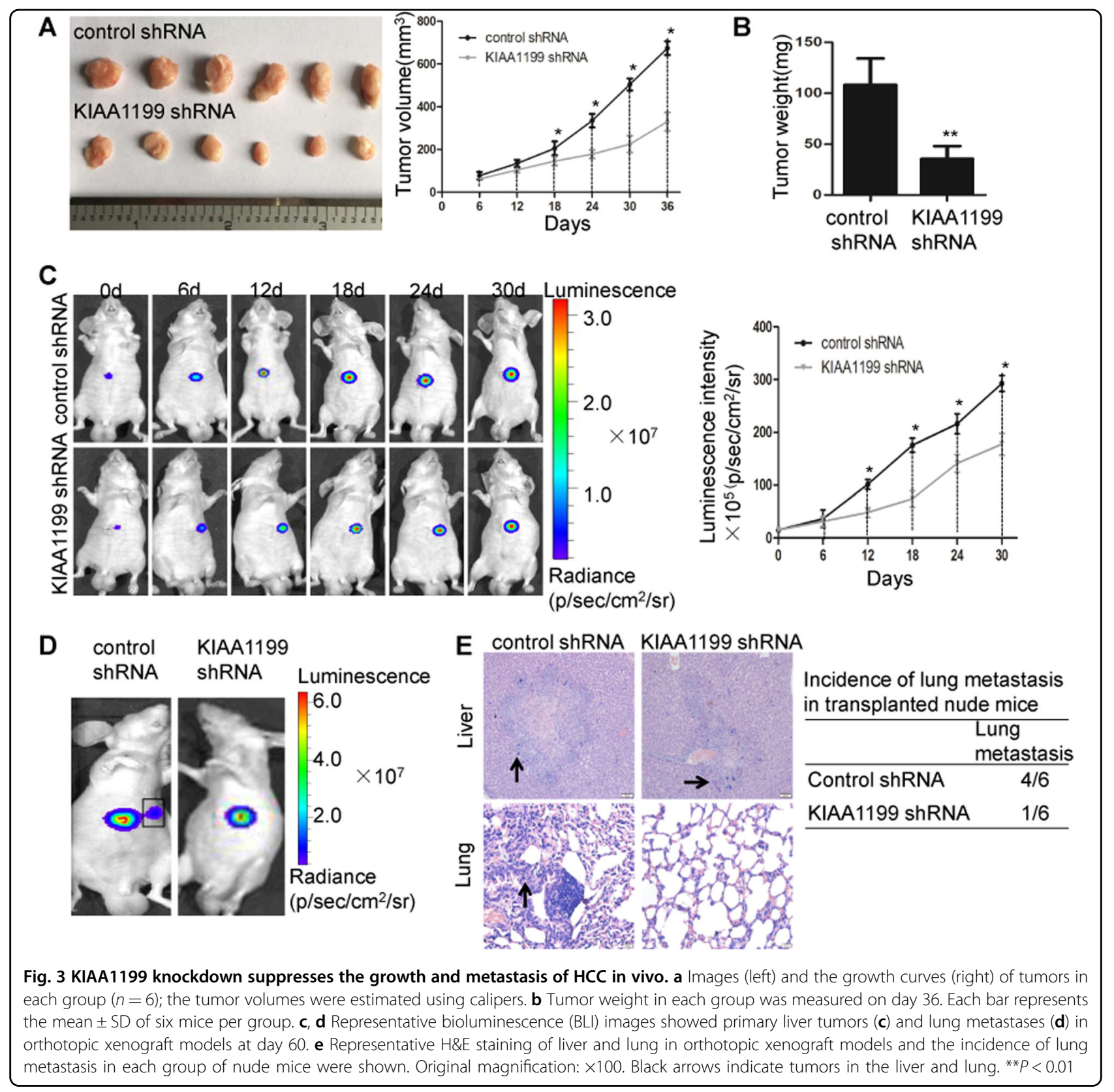

prevention of apoptosis (Fig. 5d, e). Our data also demonstrated that transfection with ATF4 siRNA and CHOP siRNA significantly attenuated cell apoptosis induced by KIAA1199 knockdown (Fig. 5f). Moreover, the TUNEL-positive cells and the caspase-3 activity increased by KIAA1199 knockdown were decreased by siATF4 and siCHOP (Fig. 5g, h). Taken together, KIAA1199 knockdown induced ER stress-mediated apoptosis in HCC cells.

\section{Discussion}

Although upregulation of KIAA1199 has been observed in various types of human cancer, such as breast cancer, gastric cancer, and colon cancer $5,7,13,14$, the role of KIAA1199 in HCC still remains unknown. In this study, for the first time, we found that KIAA1199 was significantly overexpressed in HCC tissues, especially in those with lager tumor size and metastasis. Moreover, KIAA1199 expression was positively correlated with the metastatic potential of $\mathrm{HCC}$ cells. Knockdown of KIAA1199 attenuated HCC growth and metastasis, which was supported by both in vitro and in vivo experiments. Our findings reveal that KIAA1199 may serve as a novel oncogene in the pathogenesis and progression of $\mathrm{HCC}$.

It is well-known that uncontrolled cell proliferation is the key mechanism for the progression of human 


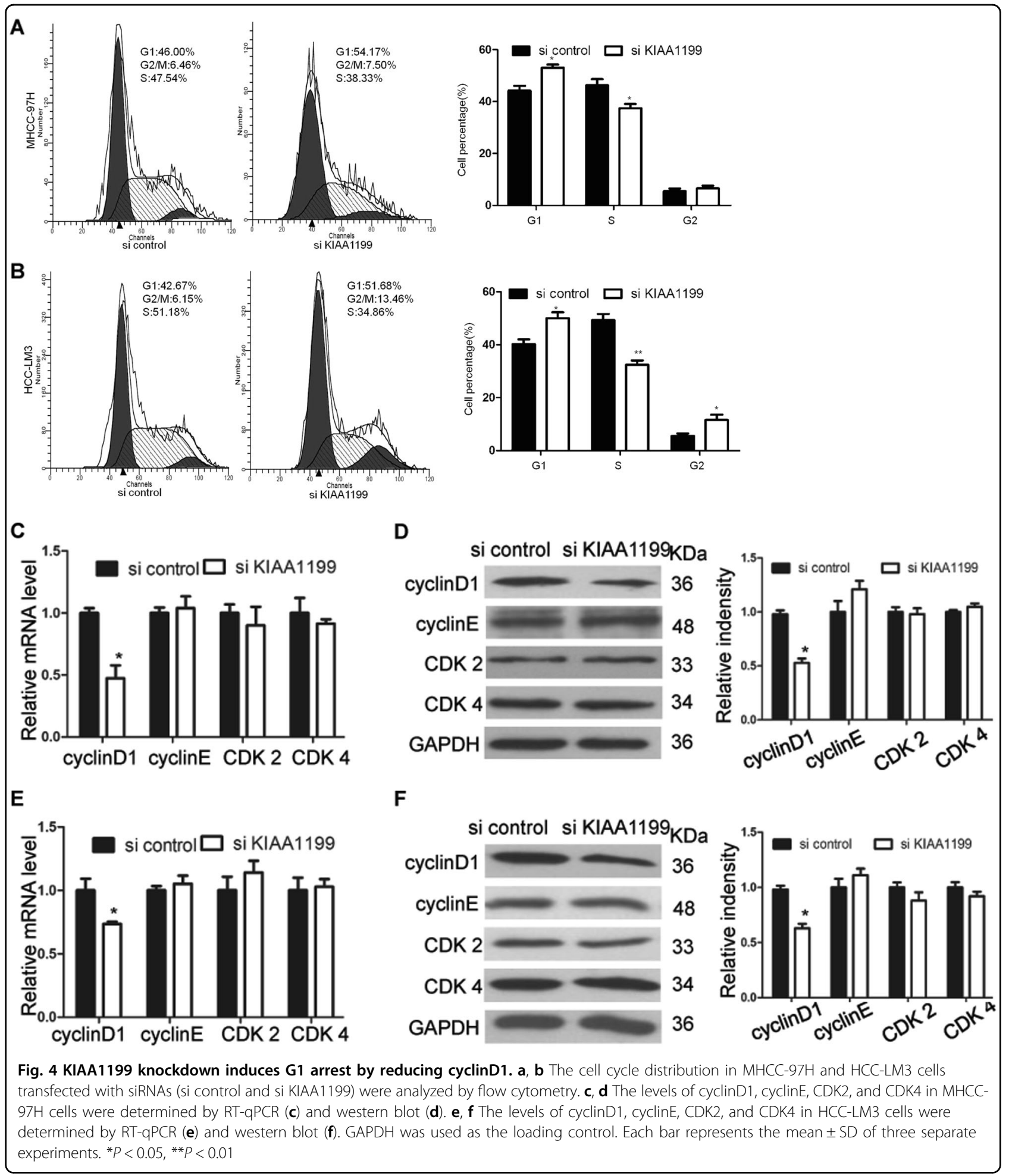

malignancies ${ }^{15}$. Birkenkamp-Demtroder et al. reported that repression of KIAA1199 decreased the proliferation of colon cancer cell line SW480 5 . Our results indicated that KIAA1199 knockdown significantly inhibited HCC cell proliferation. This was further supported by the result that silencing of KIAA1199 inhibited the growth of xenograft tumors in mice. To explore the underlying molecular mechanism, we focused on the cell cycle. Interestingly, suppression of KIAA1199 induced cell cycle arrest at G1 phase, and the expression of cyclinD1, a 


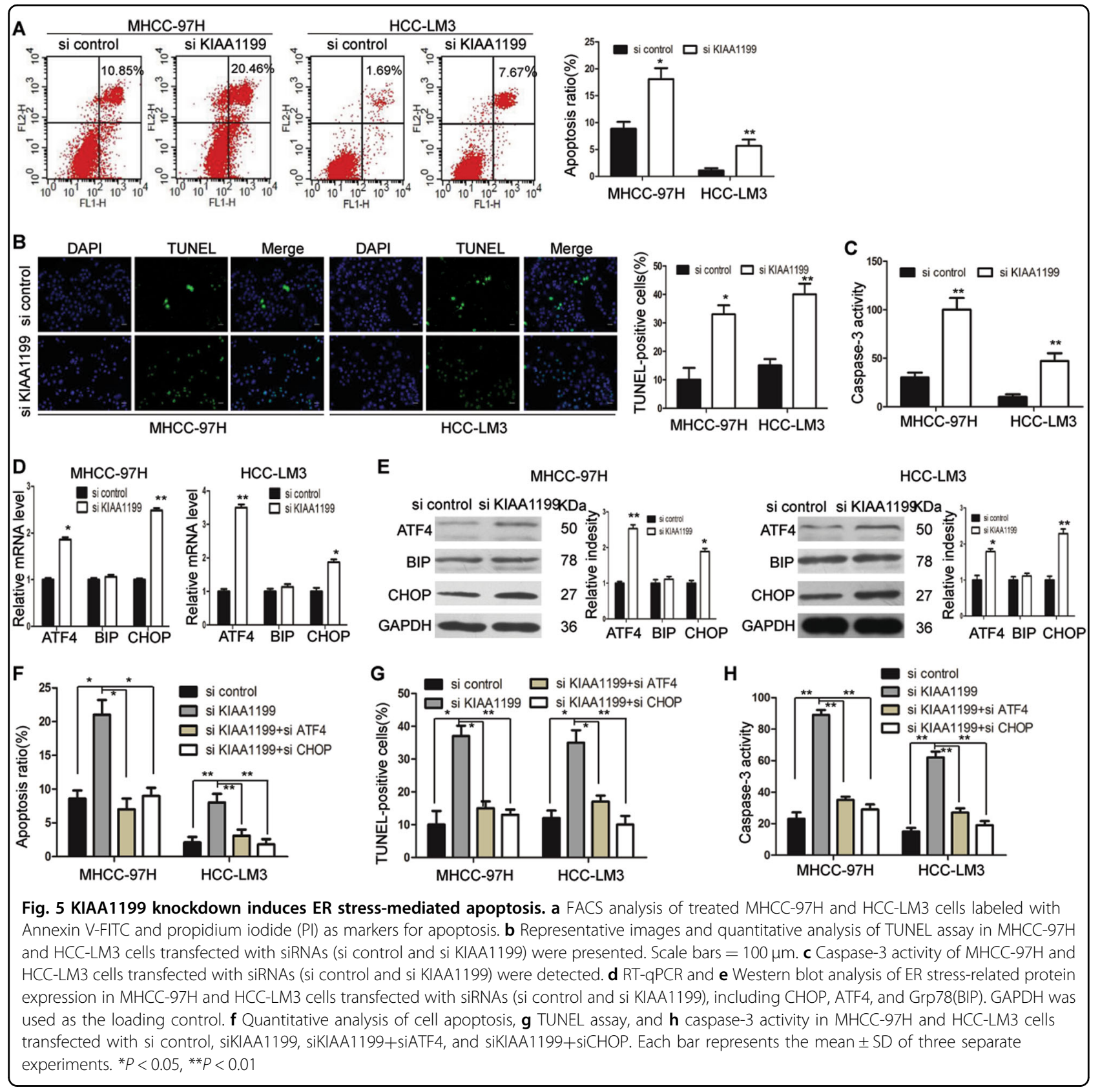

crucial regulator in cell cycle arrest, was downregulated by KIAA1199 knockdown in HCC cells.

An essential hallmark of cancer cells is the ability to evade apoptosis ${ }^{16}$. As we know, moderate ER stress can act as a protective mechanism, however, prolonged and excessive ER stress eventually leads to apoptosis ${ }^{17,18}$. Our data showed that KIAA1199 knockdown induced apoptosis in HCC cells. Furthermore, we also confirmed that reduction of KIAA1199 induced ER stress, as indicated by the upregulation of ER stress-related genes. CHOP and Grp78(BiP) are two important effectors in ER stress ${ }^{19}$. CHOP has been reported to be a crucial ER stress responsive factor that executes apoptosis, which is also a downstream target of ATF4 pathway. Grp78(BiP), an ER stress chaperone molecule, is upregulated in ER stress ${ }^{12}$. Evensen et al. showed that KIAA1199-BiP interaction is a specific cellular event required for ER retention and cell migration in breast cancer ${ }^{9}$. However, we found that reduction of KIAA1199 increased the levels of ATF4 and CHOP, but exerted no effect on BIP expression in HCC cells. Moreover, ATF4 siRNA and CHOP siRNA attenuated KIAA1199 knockdown-induced apoptosis. This study provides important evidence that KIAA1199 knockdown induced ER stress-mediated apoptosis in 
HCC cells. The possibility of involvement of other factors in apoptosis induced by KIAA1199 knockdown could not be excluded.

In conclusion, our present study suggests that KIAA1199 is involved in the development and progression of HCC. KIAA1199 knockdown inhibited cell proliferation and migration in vitro and attenuated tumor growth and metastasis in vivo. All of these results provide a wider perspective on $\mathrm{HCC}$ treatment.

\section{Materials and methods}

HCC samples and cell lines

The 64 paired tissue specimens were obtained from HCC patients (Department of Surgery, Tongji Hospital of Tongji Medical College, Huazhong University of Science and Technology, between 2011 and 2014) by way of surgery after getting their consent. The cell lines HCCLM3, MHCC-97H, MHCC-97L, SMMC-7721, Huh7, Hep3B, and L02 (Institute of liver diseases, Tongji Hospital of Tongji Medical College, Huazhong University of Science and Technology, Wuhan, Hubei, China) were cultured in DMEM medium containing 10\% fetal calf serum (Invitrogen Gibco, Carlsbad, CA, USA) and incubated in a $5 \% \mathrm{CO}_{2}$ incubator at $37^{\circ} \mathrm{C}$. All human and animal studies were performed according to the guidelines of the Ethics Committee of the Tongji Hospital and approved in accordance with the ethical standards of the World Medical Association Declaration of Helsinki.

\section{RNA extraction and real-time RT-PCR}

Total RNA was extracted using TRIzol reagent (Invitrogen, Carlsbad, CA, USA). Reverse-transcribed complementary DNA was synthesized using the PrimeScript RT reagent kit (TaKaRa, Otsu, Japan). Real-time polymerase chain reaction was performed using SYBR Premix ExTaq (TaKaRa, Otsu, Japan) on an ABI StepOne RealTime PCR System (Applied Biosystem, Carlsbad, CA, USA). The sequences of the primers used for PCR were listed in Supplemental Table 1.

\section{RNAi and establishment of overexpressing cells}

RNAi and establishment of stable expressing cells were performed as described previously ${ }^{20}$. siRNAs were synthesized by RiboBio company (Guangzhou, China) and plasmids were synthesized by Vigenebio Company (Shandong, China), and then transfected into cells using lipofectamine 2000 (Invitrogen) according to the manufacturer's instructions. The sequence of siKIAA1199 (or shKIAA1199) was designed as follows: GATCCTTACTATGGTCTGA. ATF4 siRNA sequence was designed as follows: AGGAGCAAAACAAGACAGCATTTT. CHOP siRNA sequence was designed as follows: GAGCU CUGAUUGACCGAAUTT.

\section{Wound healing and transwell assays}

Wound healing and transwell assays were performed as described previously ${ }^{20}$.

\section{CCK-8, colony formation, and caspase- 3 activity assays}

CCK-8, colony formation, and caspase- 3 activity assays were performed as described previously ${ }^{21}$.

\section{Cell cycle and apoptosis analysis}

Cell cycle analysis kit and Annexin V-FITC apoptosis kit were purchased from BD Pharmingen (San Diego, CA, USA). For cell cycle analysis, the cells were harvested after treatment, fixed with ice-cold $70 \%$ ethanol solution, hydrolyzed with RNaseA, and stained with propidium iodide (PI) for $20 \mathrm{~min}$. For apoptosis analysis, the cells were harvested after treatment, washed twice with PBS, and resuspended in $1 \times$ binding buffer. Annexin V-FITC and PI were added to the cell preparations and then incubated for $15 \mathrm{~min}$ in the dark. Cell cycle and apoptosis analysis were performed by FACS Calibur flow cytometer (Becton Dickinson, San Diego, CA).

\section{TUNEL assay}

The apoptosis assay was measured by DeadEnd ${ }^{\mathrm{mm}}$ Fluorometric TUNEL System (Promega, Madison, WI). After $48 \mathrm{~h}$ of transfection with siRNAs or plasmids, TUNEL assay was performed according to the manufacturer's instructions. Fluorescent images were captured using a Zeiss fluorescence microscope. For quantitative results, the TUNEL-positive cells were counted in five random area images for each sample by ImageJ.

\section{Western blotting and immunohistochemistry}

For western blotting, proteins were separated on SDSPAGE and transferred to nitrocellulose membrane (BioRad). The membrane was blocked with 5\% non-fat milk and incubated with the corresponding antibodies. Informations of the antibodies are listed in the Supplementary Table 2.

For immunohistochemistry, paraffin-embedded tissues were cut into 4- $\mu \mathrm{m}$-thick consecutive sections. Antigen retrieval was performed following the standard procedure. Sections were cooled and immersed in a $0.3 \%$ hydrogen peroxide solution to block the endogenous peroxidase activity. Non-specific labeling was blocked with $5 \%$ nonfat milk, and then incubated with corresponding antibody, developed by peroxidase-conjugated streptavidin and $\mathrm{DAB}$, and counterstained by hematoxylin.

\section{In vivo tumor growth and metastasis assays}

For subcutaneous xenograft models, $5 \times 10^{6}$ LM3shRNA or LM3-shKIAA1199 cells in $0.1 \mathrm{ml}$ PBS were injected into nude mice subcutaneously. The length $(L)$ 
and width $(W)$ of the tumors were measured with digital vernier calipers. Tumor volume $(T V)$ was determined according to the formula: $T V=\left(L \times W^{2}\right) 2$. For orthotopic xenograft models, $1 \times 10^{6}$ cells in $0.2 \mathrm{ml}$ PBS were injected into the subcutaneous region of nude mice. Subcutaneous tumors were harvested once reached about $10 \mathrm{~mm}^{3}$, and then cut into $1.0-\mathrm{mm}^{3}$ pieces. One piece of tumor was implanted into the left liver lobes of the nude mice. Mice were sacrificed on day 60 . Liver and lung tissues were resected and fixed with $4 \%$ paraformaldehyde, and then stained with H\&E.

\section{Statistic analyses}

All experiments in vitro were performed in triplicate unless specified. Results are represented as the mean \pm SD. KIAA1199 expression was compared with demographic and biological parameters by $\chi^{2}$ test. Statistical analysis was performed using Student's $t$ test. The $P$ values $<0.05$ were considered significant.

\section{Acknowledgements}

This study was supported by the National Natural Science Foundation of China [No. 81572419, 81672392].

\section{Conflict of interest}

The authors declare that they have no conflict of interest.

\section{Publisher's note \\ Springer Nature remains neutral with regard to jurisdictional claims in published maps and institutional affiliations.}

The online version of this article (https://doi.org/10.1038/s41420-018-0099-5) contains supplementary material, which is available to authorized users.

Received: 8 February 2018 Revised: 31 March 2018 Accepted: 8 May 2018 Published online: 12 November 2018

\section{References}

1. Torre, L. A. et al. Global cancer statistics, 2012. CA Cancer J. Clin. 65, 87-108 (2015).
2. Valastyan, S. \& Weinberg, R. A. Tumor metastasis: molecular insights and evolving paradigms. Cell 147, 275-292 (2011).

3. Liu, M., Jiang, L. \& Guan, X. Y. The genetic and epigenetic alterations in human hepatocellular carcinoma: a recent update. Protein \& Cell 5, 673-691 (2014).

4. Abe, S., Usami, S. \& Nakamura, Y. Mutations in the gene encoding KIAA1199 protein, an inner-ear protein expressed in Deiters' cells and the fibrocytes, as the cause of nonsyndromic hearing loss. J. Hum. Genet 48, 564-570 (2003).

5. Birkenkamp-Demtroder, K. et al. Repression of KIAA1199 attenuates Wntsignalling and decreases the proliferation of colon cancer cells. Br. J. Cancer 105, 552-561 (2011).

6. Kuscu, C. et al. Transcriptional and epigenetic regulation of KIAA1199 gene expression in human breast cancer. PLOS ONE 7, e44661 (2012).

7. Matsuzaki, S. et al. Clinicopathologic significance of KIAA1199 overexpression in human gastric cancer. Ann. Surg. Oncol. 16, 2042-2051 (2009).

8. LaPointe, L. C. et al. Discovery and validation of molecular biomarkers for colorectal adenomas and cancer with application to blood testing. PLOS ONE 7, e29059 (2012).

9. Evensen, N. A. et al. Unraveling the role of KIAA1199, a novel endoplasmic reticulum protein, in cancer cell migration. J. Natl. Cancer Inst. 105, 1402-1416 (2013).

10. Tiwari, A. et al. Early insights into the function of KIAA1199, a markedly overexpressed protein in human colorectal tumors. PLOS ONE 8, e69473 (2013).

11. Puthalakath, $\mathrm{H}$. et al. ER stress triggers apoptosis by activating $\mathrm{BH} 3$-only protein Bim. Cell 129, 1337-1349 (2007).

12. Verfaillie, T., Garg, A. D. \& Agostinis, P. Targeting ER stress induced apoptosis and inflammation in cancer. Cancer Lett. 332, 249-264 (2013).

13. Jami, M. S. et al. Functional proteomic analysis reveals the involvement of KIAA1199 in breast cancer growth, motility and invasiveness. BMC Cancer 14, 194 (2014).

14. Zhang, Y., Jia, S. \& Jiang, W. G. KIAA1199 and its biological role in human cancer and cancer cells (review). Oncol. Rep. 31, 1503-1508 (2014).

15. Evan, G. I. \& Vousden, K. H. Proliferation, cell cycle and apoptosis in cancer Nature 411, 342-348 (2001)

16. Hanahan, D. \& Weinberg, R. A. Hallmarks of cancer: the next generation. Cell 144, 646-674 (2011).

17. Yadav, R. K., Chae, S. W., Kim, H. R. \& Chae, H. J. Endoplasmic reticulum stress and cancer. J. Cancer Prev. 19, 75-88 (2014).

18. Tabas, I. \& Ron, D. Integrating the mechanisms of apoptosis induced by endoplasmic reticulum stress. Nat. Cell Biol. 13, 184-190 (2011).

19. Mei, Y., Thompson, M. D., Cohen, R. A. \& Tong, X. Endoplasmic Reticulum Stress and Related Pathological Processes. J. Pharmacol. \& Biomed. Anal. 1, 1000107 (2013).

20. Liu, J. et al. The histidine-rich calcium binding protein (HRC) promotes tumor metastasis in hepatocellular carcinoma and is upregulated by SATB1. Oncotarget 6, 6811-6824 (2015)

21. Liu, J. et al. Histidine-rich calcium binding protein promotes growth of hepatocellular carcinoma in vitro and in vivo. Cancer Sci. 106, 1288-1295 (2015). 